\title{
Force- and Vision-based Detection of Contact State Transitions
}

\author{
Frank ABEGG, Axel REMDE, and Dominik HENRICH
}

\begin{abstract}
A new and systematic basic approach to force- and vision-based robot manipulation of deformable (non-rigid) linear objects is introduced. This approach reduces the computational needs by using a simple state-oriented model of the objects. These states describe the relation between the deformable and rigid obstacles, and are derived from the object image and its features. We give an enumeration of possible contact states and discuss the main characteristics of each state. We investigate the performance of robust transitions between the contact states and derive criteria and conditions for each of the states and for two sensor systems, i.e. a vision sensor and a force/torque sensor. This results in a new and task-independent approach in regarding the handling of deformable objects and in a sensor-based implementation of manipulation primitives for industrial robots. Thus, the usage of sensor processing is an appropriate solution for our problem. Finally, we apply the concept of contact states and state transitions to the description of a typical assembly task. Experimental results show the feasibility of our approach: A robot performs several contact state transitions which can be combined for solving a more complex task.
\end{abstract}

\section{Introduction}

The manipulation of rigid objects by robots has been the subject of study for several decades. Less effort has been made in investigating the manipulation of nonrigid or deformable objects, despite its significance in many industrial applications [1]. Here, we focus on the one-dimensional or linear deformable objects, such as 
cables, wires, ropes, strings, beams, etc. This task has various application fields, e.g., hot-wire maintenance [2], cable form assembly, and production of control cabinets. The main problem of manipulating these objects is that they may change their shape during manipulation.

To cope with this problem, one approach is to estimate the shape of the deformable objects by calculating an internal model and simulating the object behavior. A static model of the objects and the obstacles can be calculated in two [3] or three [4] dimensions. An extension leads to a dynamic model of deformable linear objects [5]. On the one hand, the object shape can be calculated with these methods precisely (direct simulation problem). On the other hand, it is not clear how to use the object models to control the robot motion, that is, to solve the inverse simulation problem [6]. Additionally, the shape calculation can be very time consuming.

Another approach is to employ sensor systems to detect the object's shape. Vision systems can be used, for example, to guide the robot motion while the robot is making a knot with a rope [7], or to detect the shape of a flexible beam while inserting it into a hole [8, 9]. Similar to model-based approaches, the vision-based approaches are quantitative ways to measure or calculate the shape. Force/torque sensors measure forces acting on the deformable object and can be used to detect the buckling of the object when being inserted into a hole $[9,10]$.

All known approaches to vision-guided handling of deformable linear objects so far only present a solution for one special problem. In $[11,9,12]$, a flexible beam is inserted into a hole. Nakagaki et al. additionally integrate a force/torque sensor while Chen et al. only use off-line sensor processing. In [7], a highly plastically deformable rope is used which is always hanging down and not deformed by a contact with an obstacle. Byun and Nagata compute the 3D pose of deformable objects but they have to deal with the stereo correspondence problem since they use a stereo vision approach [1]. In the work of Smith, highly plastically deformable ropes are laid along a desired shape in a plane [13], but non-elastic materials like ropes are not regarded in this work so far.

A qualitative sensor-based approach to manipulate deformable linear objects is skill-based manipulation. Manipulation skills are motion primitives for achieving a particular target state of the manipulated object. They are specified in the task domain independently of the robot hardware, and hide control procedures and sensor feedback from the programmer. Skills are robust and overcome residual errors and uncertainties in both, models and manipulator movements. For example, for rigid polyhedral objects, the manipulation skills serve as transitions between contact states, and they simplify programming for a model-based manipulation system [14]. The sequence of manipulation skills can then be extracted automatically from the motion performed by an operator in a simulator [15].

The basic precondition of skill-based manipulation is the identification of object states. Then, the manipulation skills can serve as transitions between these states. The question is what kind of state models are appropriate for deformable objects. Topological states, such as provided by the knot theory, use the number and kind of crossings of the linear object (with itself) [16]. For rigid objects, contact states 
differ in the involved type and number of geometric primitives [17]. Shape states are determined by calculating the precise or approximate object geometry [4, 18] and can hardly be distinguished in a symbolic way. Position states use the location and orientation of geometric primitives relative to other geometric primitives [19].

In this chapter, we formulate a general approach for the sensor-based manipulation of deformable linear objects (DLO). We investigate contact states and point contacts of deformable linear objects and show how our approach is used to implement task-independent manipulation primitives for industrial robots. These primitives are also referred to as skills and can be combined for solving different tasks.

For solving manipulation tasks with deformable objects, we need to answer the following questions: what are the possible contact states of deformable linear objects and how do deformable linear objects in contact behave qualitatively? How does a human recognize contact state transitions? How can the approach be used together with a sensor processing system for guiding a robotic handling system by observing the changes of the object shape? What are the results of using our approach? What are the conclusions and how should the work be continued and improved?

\section{Contact State Transitions}

We introduce contact states and contact state transitions of deformable linear objects with respect to an obstacle environment. Our approach of sensor-based operations bases on the recognition of them.

\subsection{Contact states}

In the following, the contact of a deformable linear object (called workpiece) in a static environment (called obstacle) is regarded. We develop the approach based on the following two assumptions:

The first, the material of the workpiece is isotrop and homogeneous. The workpiece is assumed to be uniformly curved, that is, it is either uniformly convex or concave. The deformation caused by gravity and contact forces is elastic, that is, the deformation disappears when the stress is released. ${ }^{1}$ Example workpieces are a (short) hose or a piece of spring steal. The linear workpiece is gripped at one end and the robot gripper may perform arbitrary linear motions.

The second, all obstacles consist of convex polyhedrons. The friction between workpiece and obstacle is negligibly low. We begin our consideration with a single contact between workpiece and obstacle.

Based on the geometric primitives of DLO and obstacle, a set of contact states that enumerates all possible contact situations is derived [20]. For polyhedral

1 The workpieces belong to the object classes $\{\mathrm{E}-, \mathrm{E}+\}$ introduced by [20]. 
objects, the geometric primitives include vertices $(V)$, edges $(E)$, and faces $(F)$. The linear workpiece has two vertices and one edge between the two vertices. We name the contact states by the contact primitive of the workpiece followed by the contact primitive of the obstacle, for example V/F for vertex-face contact (Figure 1). An additional state is $\mathrm{N}$ which indicates that workpiece and obstacle are not in contact.

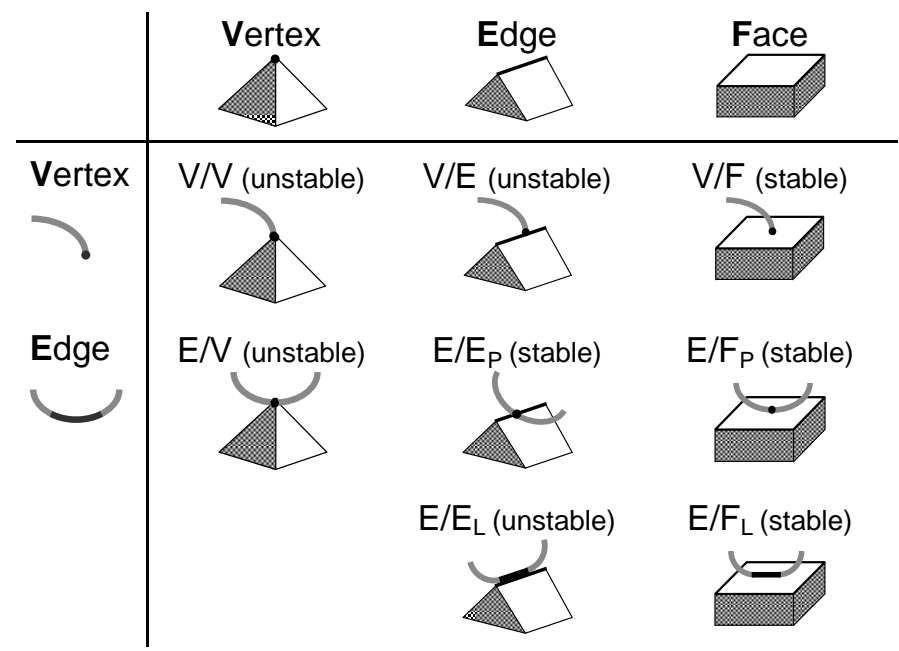

Figure 1: Enumeration of contact states between a deformable linear object and a convex polyhedron

An important attribute of each contact state is its stability. A contact state which remains unchanged as the robot gripper makes a (small) motion in any direction is called stable. However, the contact point or contact line may move. If this condition is not fulfilled, we call the contact state unstable. Consequently, a stable contact state is especially kept up when the robot gripper is not moved.

\subsection{State transitions}

State transitions are a change from one contact state to another without passing intermediate states. For now, establishing a second contact without loosening the first one, i.e., establishing a double contact, is not considered. Combining the contact states with the transitions between them, the graph shown in Figure 2 is obtained. This graph gives all possible transitions between the contact states (including state $\mathrm{N}$ ) and is found by means of basic manipulation experiments. The contact states represent nodes while the state transitions represent edges with initiated transitions (plain arrows) and spontaneous transitions (dashed arrows). Solid edges starting and ending at the same node indicate stable contact states, i.e., these states can be their own successors. This is also the case when a motion of the robot gripper is not large enough to cause a state transition. 
As stated in [21], any stable state may be directly established from state N. Stable states are connected with state $\mathrm{N}$ by solid edges. Transitions between stable states are reversible by just performing the same gripper motion in the reverse direction. For transitions beginning with unstable states, things are different. It is found that they are only partly reversible. The dashed edges in the transition graph starting from these contact states indicate that there are several possible stable successors for each of them. While transitions leading to a stable state different from $N$ (e.g. $V / F \rightarrow V / E \rightarrow V / F$ ) are reversible, those transitions leading to $N$ are irreversible [21].

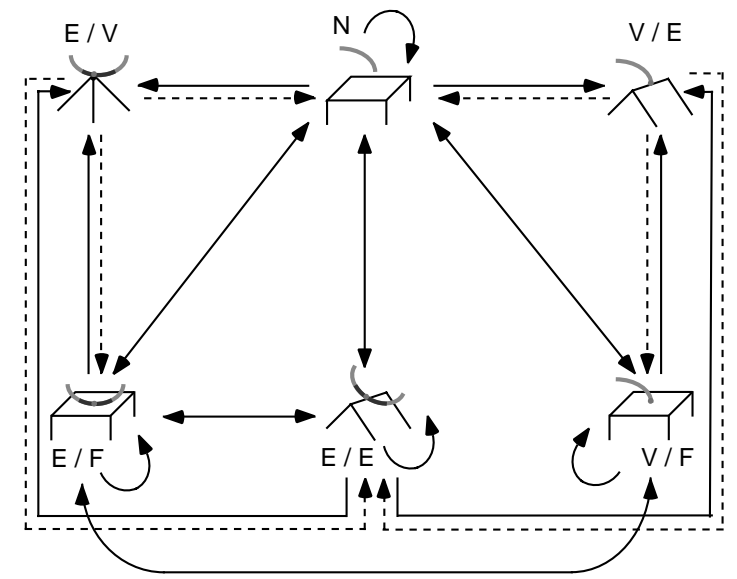

Figure 2: Contact state transition graph [21] with initiated transitions (plain arrows) and spontaneous transitions (dashed arrows)

\subsection{Programming of manipulation tasks}

So far, we have presented contacts of a deformable linear workpiece with a rigid obstacle and contact state transitions from an analytical point of view. This section introduces our concept to the application of assembly tasks.

As far as rigid workpieces are concerned, a lot of works address the problem of developing robust and flexible routines for typical assembling or disassembling tasks. The basic idea is to set up a library of encapsulated, sensor-based routines that can be used as a construction kit for efficiently solving complex assembly problems. Morrow and Khosla demonstrate the efficiency of this method in inserting different kinds of plug-in connectors [19].

Morris shows that performing assembly tasks can be regarded as a stepwise increasing of the number of constraints (reducing the degrees of freedom) of one of the mating parts by establishing contact with the other part [22]. Therefore, detecting and manipulating the contact state of the mating parts is a key issue for developing manipulation routines. Any routine that changes the contact state of the mating parts, like establishing point contact, transferring point contact to face contact, etc., forms a module of the construction kit for assembly operations. 
When thinking of those tasks, it is found that the basic assumptions on our working environment must be partly relaxed. This affects especially the assumptions of one single contact and of a convex polyhedral obstacle. ${ }^{2}$ However, this does neither affect the applicability of the set of contact states nor the rules derived for state transitions.

Let us consider the problem of inserting an elastic pneumatic hose into an Ushaped guiding groove as shown in Figure 3.
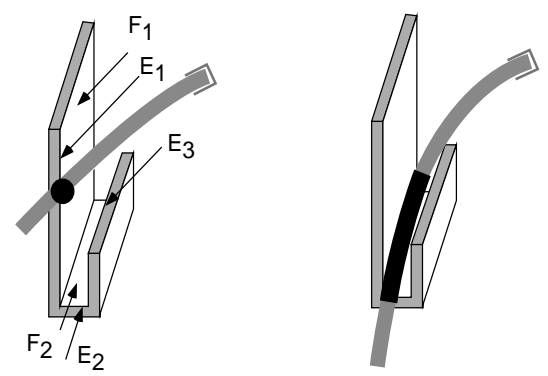

Figure 3: Two states of the insertion process of a hose into an U-shaped guiding groove

Depending on the boundary conditions, there are several possibilities to perform this task. A rather simple and robust procedure is as follows:

- Establish an initial contact between the edge of the hose and edge $\mathrm{E}_{1}$ of the groove.

- Move the hose downwards without loosening contact until the hose touches either one of the edges $\mathrm{E}_{2}$ or $\mathrm{E}_{3}$.

- $\quad$ Establish contact between the hose edge and $F_{1}$.

- Move the hose downwards without loosening contact with $\mathrm{F}_{1}$ until it gets in contact with $\mathrm{F}_{2}$.

Translating this procedure into a sequence of contact states and state transitions leads to the following:

$$
\begin{gathered}
\mathrm{N} \rightarrow \mathrm{E} / \mathrm{E}_{1} \rightarrow \mathrm{E} / \mathrm{E}_{1} \wedge\left(\mathrm{E} / \mathrm{E}_{2} \vee \mathrm{E} / \mathrm{E}_{3}\right) \\
\rightarrow \mathrm{E} / \mathrm{F}_{1} \rightarrow \mathrm{E} / \mathrm{F}_{1} \wedge \mathrm{E} / \mathrm{F}_{2}
\end{gathered}
$$

Obviously, it would be rather simple to generate a robot program to perform this task if a library of sensor-based, encapsulated routines for the required state transitions was available. Performing assembly tasks in this way requires neither exact knowledge about the mechanical workpiece properties nor a quantitative calculation of the workpiece shape.

2 The occurrence of a second contact is needed as a trigger signal for initiating a new gripper motion. However, the further behavior of the second contacts is not relevant for performing the task. 


\section{Human Transition Recognition}

Based on the ideas introduced above, several experiments are performed in order to gain knowledge about the behaviour of the deformable object and to detect contact state transitions of the workpiece. At first, a list of features is derived from the manual manipulation experiments. Secondly, characteristic measurement curves are generated by observing the features during a state transition. The manual manipulation experiments are verified with a robot and two technical sensor systems in the next section.

\subsection{Sensor data features}

In the first experiment, a human grips a low elastic workpiece, which belongs to the material class E- defined in [20], for example a pneumatic wire. Those workpieces are deformed elastically when the deforming force is greater than gravity. As obstacles for the manipulation, cubic and pyramidal objects are used similar to the objects shown in Figure 2. Then, each transition of the graph between two stable states with one unstable state in between is examined by a human simulating some sensors, which are in our case visual sensors (a stationary camera with several different viewpoints and a hand camera) and a force/torque sensor. The observed features are one-dimensional values that are directly measured by the sensor or derived from those low-level data. Examples are one single coordinate of the free workpiece endpoint or the angle of the tangent in this point.

\subsection{Characteristic functions}

By observing manually initiated state transitions, we find that the change of the values of the (one-dimensional) features always can be assigned either to a characteristic feature change description $\mathrm{L}(a, b, c, d)$ or to a description $\mathrm{P}(a, b, c, d)$ which are both defined in Table $1 . \mathrm{L}(a, b, c, d)$ is a useful description when it can be assumed that the curve of feature values is piecewise linear. This is found to be true for slow workpiece motions, for smoothing of the values, and when there is no sudden stress release.

\begin{tabular}{llll}
\hline Type & Parameter & Condition \\
\hline $\mathrm{L}$ & $a:=$ & $\operatorname{sign}\left(\lim _{t \rightarrow t_{0}+} f(t)-\lim _{t \rightarrow t_{0}-} f(t)\right)$ \\
& $b:=$ & $\operatorname{sign}\left(\lim _{t \rightarrow t_{0}-} \Delta f(t)\right)$ \\
& $c:=$ & $\operatorname{sign}\left(\lim _{t \rightarrow t_{0}+} \Delta f(t)\right)$ \\
& $d:=$ & $\operatorname{sign}\left(\lim _{t \rightarrow t_{0}+} \Delta f(t)-\lim _{t \rightarrow t_{0}-} \Delta f(t)\right)$ \\
\hline $\mathrm{P}$ & $a:=$ & $f\left(t \leq t_{0}\right)$ \\
& $b, c:$ & $f\left(t>t_{0}\right)=f\left(t_{0}\right)+b \sin (\omega t) e^{-c t}$ \\
\hline
\end{tabular}

Table 1: Definition of the parameters $\mathrm{L}(a, b, c, d)$ and $\mathrm{P}(a, b, c)$ characterizing the changes in the curves of the feature values 
Note that $a, b, c, d$ are defined as elements of the set $\{-1,0,+1\}$. If there is a sudden stress release and oscillations in the workpiece occur, $\mathrm{P}(a, b, c)$ can be used to describe the feature value curve. Examples for some characteristic curves are provided in Figure 4.
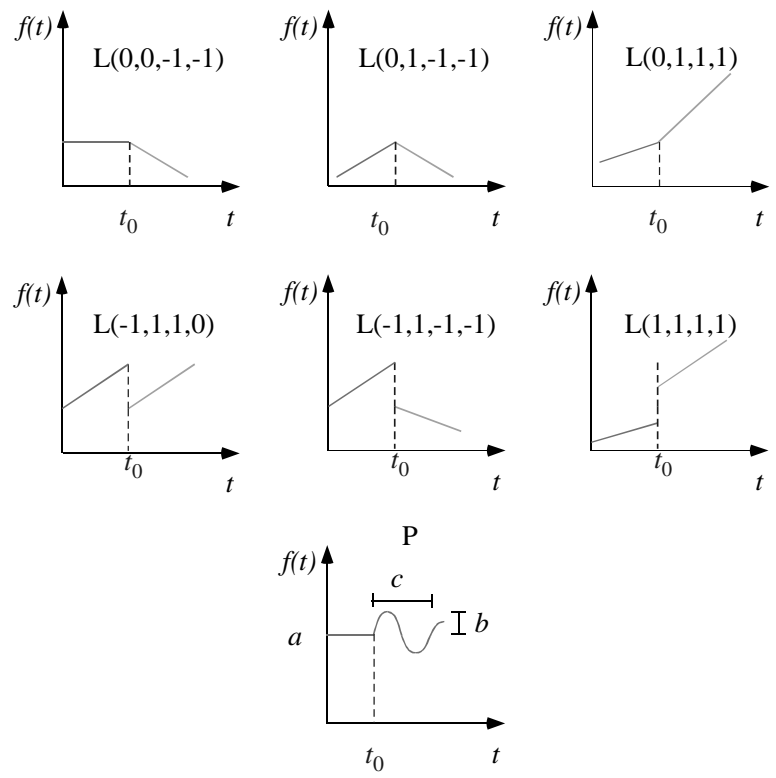

Figure 4: Examples for the observed characteristic changes of features $f(t)$. $\mathrm{L}(0,0,-1,-1)$ : change after constancy, $\mathrm{L}(0,1,-1,-1)$ : roof/valley, $\mathrm{L}(0,1,1,1)$ : slope change, some jumps $\mathrm{L}( \pm 1, \ldots)$, and the general look of a curve of type $\mathrm{P}(a, b, c)$

\subsection{Human observation}

One result of the manual experiments concerning the vision sensor is shown in Table 2. In the table, all state transitions that occur between two stable states are classified according to the four visual features curvature $u(t)$, endpoint $p(t)$, tangent angle in the endpoint $g(t)$, angle between endpoints $n(t)$. Due to the simulation of low sample rates, oscillations that would result in a $\mathrm{P}(a, b, c)$-curve are not observed.

For the theoretical force/torque analysis we assume an ideal force/torque sensor, i.e., a sensor with infinite resolution and without internal noise. Furthermore, we do not consider friction. ${ }^{3}$ In a first practical validation, where the tactile sense of a human hand joint is used as a substitute for a wrist-mounted technical force/torque

3 Some additional assumptions concerning the contact state transitions which are of minor importance here are given in [21]. 
sensor, we find all the classes of feedback curves defined in Figure 4. Similar results as in Table 2 can be expected.

\begin{tabular}{lllll}
\hline & \multicolumn{4}{l}{ Characteristic feature change for stationary vision observation } \\
Transition & $u(t)$ & $p(t)$ & $g(t)$ & $n(t)$ \\
\hline $\mathrm{N} \rightarrow \mathrm{V} / \mathrm{F}$ & $(0,0, c, d)$ & $(0, \pm 1,0, \pm 1)$ & $(0,0, c, d)$ & $(0,0, c, d)$ \\
$\mathrm{N} \rightarrow \mathrm{E} / \mathrm{E}$ & $(0,0, c, d)$ & $(0, \pm 1, \pm 1, \pm 1)$ & $(0,0, c, \pm 1)$ & $(0,0, c, \pm 1)$ \\
$\mathrm{N} \rightarrow \mathrm{E} / \mathrm{F}$ & $(0,0, c, d)$ & $(0, \pm 1, \pm 1, \pm 1)$ & $(0,0, \pm 1, \pm 1)$ & $(0,0,1, \pm 1)$ \\
$\mathrm{V} / \mathrm{F} \rightarrow \mathrm{N}$ & $(0, \pm 1,0, \pm 1)$ & $(0,0, c, \mathrm{~d})$ & $(0, \pm 1,0, \pm 1)$ & $(0, \pm 1,0, \pm 1)$ \\
$\mathrm{V} / \mathrm{F} \rightarrow \mathrm{E} / \mathrm{F}$ & $(0, \pm 1,0, \pm 1)$ & $(0,0, c, d)$ & $(0, \pm 1,0, \pm 1)$ & $(0, \pm 1,0, \pm 1)$ \\
$\mathrm{V} / \mathrm{F} \rightarrow \mathrm{V} / \mathrm{E} \rightarrow \mathrm{N}$ & $( \pm 1, b, 0, \pm 1)$ & $( \pm 1, b, c, d)$ & $( \pm 1, b, 0, \pm 1)$ & $( \pm 1, b, 0, \pm 1)$ \\
$\mathrm{V} / \mathrm{F} \rightarrow \mathrm{V} / \mathrm{E} \rightarrow \mathrm{V} / \mathrm{F}$ & $(0, \pm 1, \pm 1, \pm 1)$ & $(0, \pm 1, \pm 1, \pm 1)$ & $(0, \pm 1, \pm 1, \pm 1)$ & $(0, \pm 1, \pm 1, \pm 1)$ \\
$\mathrm{V} / \mathrm{F} \rightarrow \mathrm{V} / \mathrm{E} \rightarrow \mathrm{E} / \mathrm{E}$ & $(0, \pm 1, \pm 1, \pm 1)$ & $(0, \pm 1, \pm 1, \pm 1)$ & $(0, \pm 1, \pm 1, \pm 1)$ & $(0, \pm 1, \pm 1, \pm 1)$ \\
$\mathrm{E} / \mathrm{E} \rightarrow \mathrm{N}$ & $(0, \pm 1,0, \pm 1)$ & $(0, \pm 1, \pm 1, \pm 1)$ & $(0, \pm 1,0, \pm 1)$ & $(0, \pm 1,0, \pm 1)$ \\
$\mathrm{E} / \mathrm{E} \rightarrow \mathrm{E} / \mathrm{F}$ & $(0, \pm 1,0, \pm 1)$ & $(0, \pm 1,0, \pm 1)$ & $(0, \pm 1,0, \pm 1)$ & $(0, \pm 1,0, \pm 1)$ \\
$\mathrm{E} / \mathrm{E} \rightarrow \mathrm{V} / \mathrm{E} \rightarrow \mathrm{N}$ & $( \pm 1, b, 0, \pm 1)$ & $( \pm 1, b, c, \pm 1)$ & $( \pm 1, b, 0, \pm 1)$ & $( \pm 1, b, 0, \pm 1)$ \\
$\mathrm{E} / \mathrm{E} \rightarrow \mathrm{V} / \mathrm{E} \rightarrow \mathrm{V} / \mathrm{F}$ & $(0, \pm 1, \pm 1, \pm 1)$ & $(0, \pm 1, \pm 1, \pm 1)$ & $(0, \pm 1, \pm 1, \pm 1)$ & $(0, \pm 1, \pm 1, \pm 1)$ \\
$\mathrm{E} / \mathrm{E} \rightarrow \mathrm{E} / \mathrm{V} \rightarrow \mathrm{N}$ & $( \pm 1, b, 0, \pm 1)$ & $( \pm 1, b, c, \pm 1)$ & $( \pm 1, b, 0, \pm 1)$ & $( \pm 1, b, 0, \pm 1)$ \\
$\mathrm{E} / \mathrm{E} \rightarrow \mathrm{E} / \mathrm{V} \rightarrow \mathrm{E} / \mathrm{E}$ & $(0, b, c, \pm 1)$ & $(0, b, c, \pm 1)$ & $(0, b, c, \pm 1)$ & $(0, b, c, \pm 1)$ \\
$\mathrm{E} / \mathrm{E} \rightarrow \mathrm{E} / \mathrm{V} \rightarrow \mathrm{E} / \mathrm{F}$ & $(0, b, c, \pm 1)$ & $(0, b, c, \pm 1)$ & $(0, b, c, \pm 1)$ & $(0, b, c, \pm 1)$ \\
$\mathrm{E} / \mathrm{F} \rightarrow \mathrm{N}$ & $(0, \pm 1,0, \pm 1)$ & $(0, \pm 1, \pm 1, \pm 1)$ & $(0, \pm 1,0, \pm 1)$ & $(0, \pm 1,0, \pm 1)$ \\
$\mathrm{E} / \mathrm{F} \rightarrow \mathrm{V} / \mathrm{F}$ & $(0, b, b, 0)$ & $(0, \pm 1,0, \pm 1)$ & $(0, b, b, 0)$ & $(0, b, b, 0)$ \\
$\mathrm{E} / \mathrm{F} \rightarrow \mathrm{E} / \mathrm{E}$ & $(0,0, \pm 1, \pm 1)$ & $(0,0, \pm 1, \pm 1)$ & $(0,0, \pm 1, \pm 1)$ & $(0,0, \pm 1, \pm 1)$ \\
$\mathrm{E} / \mathrm{F} \rightarrow \mathrm{E} / \rightarrow \mathrm{VN}$ & $( \pm 1, b, 0, \pm 1)$ & $( \pm 1, b, c, \pm 1)$ & $( \pm 1, b, 0, \pm 1)$ & $( \pm 1, b, 0, \pm 1)$ \\
$\mathrm{E} / \mathrm{F} \rightarrow \mathrm{E} / \mathrm{V} \rightarrow \mathrm{E} / \mathrm{E}$ & $(0, b, c, d)$ & $(0, b, c, d))$ & $(0, b, c, d)$ & $(0, b, c, d)$ \\
$\mathrm{E} / \mathrm{F} \rightarrow \mathrm{E} / \mathrm{V} \rightarrow \mathrm{E} / \mathrm{F}$ & $(0, b, c, d)$ & $(0, b, c, d)$ & $(0, b, c, d)$ & $(0, b, c, d)$ \\
\hline & & & &
\end{tabular}

Table 2: Characteristic changes of four visual features for each of the contact state transitions

\subsection{Observation with technical sensors}

In the case of "real" sensor systems, we again obtain measurement value curves that can be qualitatively characterized by the classes defined in Figure 4. This holds true for all possible state transitions and special measurement values of two sensors: a CCD camera observing the DLO from a stationary viewpoint and a 6 DOF force/torque sensor mounted at the wrist of an industrial robot. The main difference with respect to the curves from the human observation is the presence of noise. Periodic and damped oscillations in the curves coming from released inner stress of the DLO are only observed if the sample rate of the sensor is high enough.

In order to see what is measured during a state transition, we need to analyze for example the initiated transition $\mathrm{N} \rightarrow \mathrm{V} / \mathrm{F}$. As similar observations are made for the 
vision system (see next Section), we only consider the case with a force/torque sensor. Let $F(t)$ and $M(t)$ be the vectors of force and moment measured by the force/torque sensor. Assuming that both force and moment are zero when the DLO is in state $\mathrm{N}$, we can observe the following: When the workpiece touches the obstacle face, it is deformed, causing force $|F|$ and moment $|M|$ rising in a linear manner. Both $|F|$ and $|M|$ increase as long as the gripper motion is continued. The same result is obtained for transitions $N \rightarrow E / E$ or $N \rightarrow E / F$. During the complete motion, $F$ is aligned with the normal of the obstacle face.

Experiments with other transitions show that any state transition is generally accompanied by a distinct change in the course of these functions [23]. Therefore, the task of detecting contact state transitions can be transferred into the problem of detecting these changes in the force and moment signals. The next section introduces our approach for the automatic transition recognition based on the automatic detection of those signal changes.

\section{Automatic Transition Recognition}

This section deals with the automatic state transition recognition that is used to perform automatically sensor-based robot operations. Again, we use the two different sensors: CCD-camera and force/torque sensor.

\subsection{General concept}

Since we believe that robust manipulation is possible without geometric reconstruction and without an exact model of the workpiece and the scene, our focus is on measuring features of the workpiece, especially the features stating any deformation in the workpiece. The deformation in the workpiece is detected by the change in several visual and force/torque features, which are extracted from the workpiece shape in the image space or the inner forces and torques resulting in a measureable stress. Since deformation can mean a change in the workpiece state in the context of one state model, changes of the measured features of the workpiece and knowledge about the initial workpiece state are used to derive the current contact state as illustrated in Figure 5. Please note that the coarse obstacle geometry is a-priori given, since it is assumed not to change and therefore can be easily assigned by the programmer or an environment data base.

In our context, sensori-motor primitives transfer a deformable workpiece from a given initial (A) stable contact state to another, desired contact state (E) with guarded robot moves: during the robot motion, the workpiece features are evaluated and used to recognize the current workpiece state. Based on the current state estimation, the robot is moved by a sequence of linear movement commands that aim to transfer the workpiece to a desired final state. Generally speaking, a sensor feature-based, sensor-driven robot control is built. The core of the control are taskindependent sensori-motor primitives which here are also referred to as manipulation skills $[24,19]$. In this work, sensori-motor primitives control the state 
of the deformable workpiece. In general, these primitives have a controller-like structure as illustrated in Figure 6 and can be combined to task dependent robot operations like such as the threading of a workpiece through a hole.

The next section describes the details of the feature extraction for the two sensors vision and and force torque sensor and a further section shows details of the recognition process.

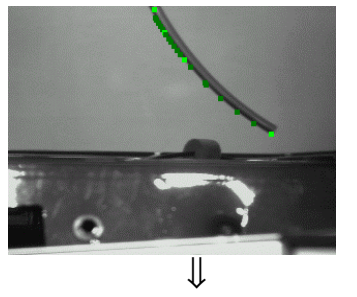

$\mathrm{N}$

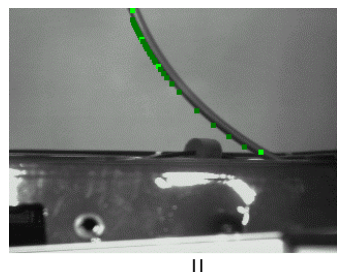

$\Downarrow$

$\mathrm{V} / \mathrm{F}$

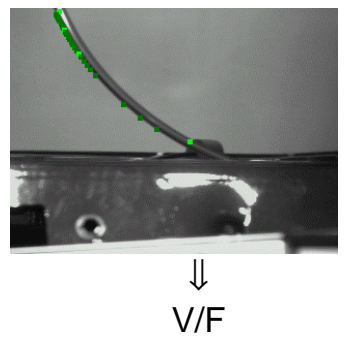

$\mathrm{V} / \mathrm{F}$

Figure 5: Recognition of workpiece states in an image sequence

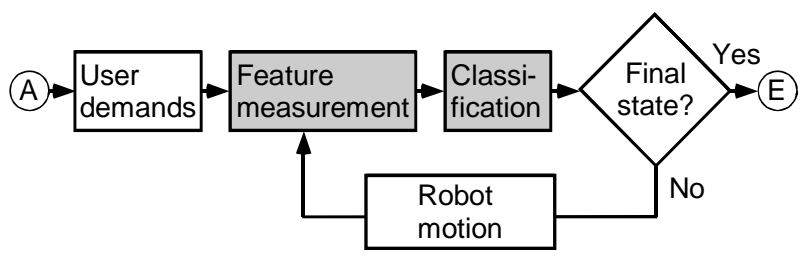

Figure 6: Structure of sensori-motor primitives with initial state $(A)$ and final state $(E)$

\subsection{Feature extraction}

This section explains how to extract the features for measuring and recognizing a transition from the sensors. At first, the visual segmentation is discussed. Secondly, the analysis of the force-torque sensor is regarded.

\subsubsection{Visual feature extraction}

Up to now, many approaches realizing different tasks of a visually guided robotic system are stated in literature [25]. However, there are only few approaches concerning deformable objects, in spite of many important industrial applications [1]. However, it is obvious that vision sensors are well-suited for observing characteristic shape changes of deformable objects. In that way, the information provided by vision sensors is complementary to that of other sensors as for example force/torque sensors. But how can the object be segmented from the image? And which image features can be used for state transition recognition of the object?

In order to get information about the shape of a deformable workpiece, it is necessary to segment the workpiece from the image background. This is commonly known as segmentation. Here, it is assumed that the workpiece is observed from a 
stationary viewpoint where characteristic shape changes occur in the image. ${ }^{4}$ After the segmentation, the deformable workpiece has to be characterized and so some features of the visual data have to be derived. Thus, we need to handle two tasks: workpiece segmentation and feature extraction.

Since there is no need to extract the obstacles from the image and a stationary camera is given, we are using a differential image method to get an idea where the main information about the deformable workpiece is. For that, the current image of the scene including the workpiece is subtracted from a reference image of the scene without workpiece. Currently, we also have some extraction algorithms for the hand camera which work without the differential image method.

Assuming a nearly constant illumination, the image information is furthermore reduced by binarizing the current differential image which results in an image where the workpiece is black and the background completely white or vice versa. The threshold for binarization is determined by taking a value near the mean gray value of the differential image. Even changing illumination is allowed if the pixels not belonging to the workpiece of the differential image are taken as new reference image pixels. After the workpiece image has been segmented, the next step is to generate a representation of the workpiece from which features can be derived which describe the deformation. In the case of linear workpieces, the curve of the projected shape is characteristic for the deformation state. Thus, data from the image of the workpiece represent the workpiece curve. Unfortunately, even thin linear workpieces produce an image with several pixels of extension. In order to get a single curve, the workpiece has to be thinned with thinning operations. But since thinning operations are time consuming iterative operations and tend to be not robust against perturbations in the image, we developed a new algorithm based on a contour following principle. The algorithm searches characteristic image points along the image border of the workpiece. The binarization has not to be made over the whole image, and thus, we save time by performing the binarization locally while searching for the contour. Further details can be found in [26].

Now the workpiece is approximated by points that lie on one borderline of its image. With little effort, it is possible to collect base points of the workpiece that have the same pixel distance. The Manhattan norm is used as distance value. Characteristic base points with different distances are already given by the corner points of the rectangles. Figure 7 shows a workpiece with detected base points. A similar but more complex algorithm has been developed for the hand camera. This algorithm computes also a base point list similar to the one for the stationary camera. Thus, the representation of the workpiece using a base point list is independent from the used sensor although some particular features derived from the base point list may behave different for the two different vision sensors.

4 Working in the image space allows us to change the camera position without needing a subsequent calibration process. 


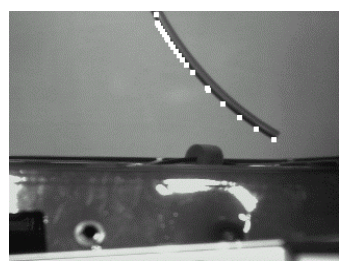

Figure 7: With the stationary camera detected pneumatic wire and found base points (white dots)

Using the base point list as a representation of the workpiece in the image, the features derived from this list give hints to the contact state of the workpiece. These hints come up during the time of robot manipulation. Thus, dynamic features are regarded. Note that the initial and final workpiece state from the graph are given by the operator.

With a list of base points of the two-dimensional image space, three types of characteristic features are considered. These features concern with (1) the start or endpoint of the list, (2) one point within the list, or (3) the whole list of base points. Experiments with our vision system show that features of type (1) and (3) give the most useful information about the workpiece state for a stationary camera as well as for a hand camera.

The following list provides examples for these types of features, which can be efficiently computed:

- $\quad$ The pixel length $l(t)$ of the workpiece. Here, the length is the sum of the lines between the base points in pixels.

- The angle $a(t)$ between the line through the two endpoints and the image axis $u$.

- $\quad$ The coordinates $\boldsymbol{p}(t)=(u(t), v(t))$ of the endpoint not gripped are used for detecting changes of the contact state of the endpoint. Changes depend on the geometric relation between the workpiece and the workpiece endpoint.

- $\quad$ The tangent angle $g(t)$ in the endpoint indicates a change of the geometric relation between endpoint and obstacle.

- The maximum curvature or the sum of curvatures where the curvature of a base point $\left(u_{i}, v_{i}\right)$ is approximated by the discretization of the curvature $k$ for regular curves $f$ according to [27]:

- $\quad k\left(u_{i}, v_{i}\right)=\frac{\Delta f\left(u_{i}, v_{i}\right) \Delta^{2} f\left(u_{i}, v_{i}\right)-\Delta^{2} f\left(u_{i}, v_{i}\right) \Delta f\left(u_{i}, v_{i}\right)}{\sqrt[3]{\Delta f^{2}\left(u_{i}, v_{i}\right)+\Delta^{2} f\left(u_{i}, v_{i}\right)}}$

The first experiments reported in the next section show the applicability of this approach for robust state transition detection, when appropriate features are combined.

Having discussed the visual feature extraction, it now shall be considered how the features for state transition detection may be extracted by means of a (non-ideal) force/torque sensor mounted at the robot wrist. 


\subsubsection{Force/torque feature extraction}

So far, we have discussed the forces caused by the interaction between DLO and obstacle. However, in most practical situations, there is a significant additional load due to gravity. As long as we restrict the gripper trajectory to translational motions, the gravity only causes a constant offset for both the force and the moment. Therefore, it does not have to be considered if absolute thresholds are avoided in the detection algorithm.

Opposite to the handling of rigid workpieces, especially two additional problems must be regarded: First, the force caused by contact with the rigid environment is generally low. This results in the need for a high sensor resolution. However, the resolution is correlated with the measuring range (A high resolution requires a small measuring range and vice versa). Therefore, the measuring range should be as small as possible. Because the force and moment caused by gravity is typically much larger than the contact force, the required measuring range (and, thus, the obtained resolution) is determined by gravity.

Secondly, all contact forces highly depend on both the workpiece and specific situation. Therefore, it is necessary to either have rather precise a priori knowledge about the workpiece and the task, or to base the transition detection on the qualitative coarse of force and moment while avoiding absolute thresholds. In order to meet the fundamental requirements given for manipulation skills, the second approach should be preferred.

As the first step of the signal processing, some filtering is required for noise reduction. In our experiments, we succeeded in using a moving average low pass filter which can be implemented very efficiently for our purpose. Below, we generally refer to $\boldsymbol{F}$ and $\boldsymbol{M}$ as filtered force and moment signals.

For evaluating the $6 \mathrm{D}$ force/moment vector, there are several possibilities. We succeeded in evaluating the absolute values of force and moment or in evaluating the six single signals independently. The directions of the vectors do not have to be considered here because it does not promise to be advantageous and the effort for a vectorial evaluation is rather high. In the following discussion of the detection algorithms, we always refer to a general function $f$, which is sampled. This may be either some visual features or a single component of $\boldsymbol{F}$ or $\boldsymbol{M}$, or the absolute values of these vectors.

\subsection{State transition recognition: detection of characteristic functions}

Now, the following two questions are investigated: how can state transitions be detected with a sensor system (vision and force/torque sensor) and what is necessary to perform the state transitions reliably and robustly with a robot system?

By observing state transitions executed by the robot and the corresponding feature values processed and analyzed by our sensor systems, we find that the change in the values of the (one-dimensional) features follows the same pattern as for the manual experiments. As a consequence, we can use them as well here. This means especially that we have to provide at least two state transition detection 
algorithms, at least one for each curve type $\mathrm{L}$ and $\mathrm{P}$.

Fortunately, through several different experiments for each the vision and force/torque sensor, we found that almost the same algorithms can be applied on both sensor systems. Only some parameters of the algorithms change depending on the sensor. The algorithm parameters are also dependent on the current environment. In the following, the two algorithms, which are still in the test phase, are described. further details can be found in [23].

\subsection{Sensor signal processing}

After the analytical discussion of the state transitions in the previous section, it shall be considered how they may be detected by means of a (non-ideal) vision sensor (stationary camera or robot hand-mounted camera) or a (non-ideal) force/torque sensor (FTS) mounted at the robot wrist. Before presenting the evaluation algorithms, some general aspects are discussed.

The detection of the $\mathbf{L}(\boldsymbol{a}, \boldsymbol{b}, \boldsymbol{c}, \boldsymbol{d})$ transitions can be traced back to the problem of detecting (more less abrupt) changes in the slope of the feature curve $f$ provided from a certain sensor. For the moment, we assume a function with always positive slope and the slope being higher for $t>t_{0}$ than for $t<t_{0}$.

The detection algorithm is based the fact that the slope $f^{\prime}(t)$ of function $f(t)$ changes rather abruptly for $t=t_{0}$. For the ideal case of two linear segments, $f^{\prime}(t)$ is a saltus function. Since this is not the case in practical applications and there is generally some noise remaining after low-pass filtering, we use the following function instead of $f^{\prime}(t)$. Let $\Delta t$ be the sampling period, and be $f(t)=f(i \Delta t)=f_{i}$ the function value sampled in the current time step $i, m_{\mathrm{MSL}},{ }_{i}$ is the slope of the mean straight line (MSL) computed from the $k+1$ samples $i-k, i-k+1, \ldots, i$ by a standard algorithm. Based on $m_{\mathrm{MSL}}$, we define

$$
\Delta m_{\mathrm{Ratio}, i}=\frac{m_{\mathrm{MSL}, i}-m_{\mathrm{MSL}, i-k}}{m_{\mathrm{MSL}, i-k}}
$$

as the relative slope change of two MSLs touching at $i_{0}$.

To clarify the characteristics of $\Delta m_{\text {Ratio, }}$, Figure 8 shows the values $f_{i}$ of a discrete function vector $f$ with slopes $m_{\text {left }}=1$ and $m_{\text {right }}=4$ together with $\Delta m_{\text {Ratio, } i \text {. The }}$ mean straight lines are computed with $k+1=21$ samples. At the beginning, $\Delta m_{\text {Ratio }}$ is 0 , i.e., both slopes in the nominator of $\Delta m_{\text {Ratio }}$ have the same value. As $i$ is approaching $i_{0}+k, \Delta m_{\text {Ratio }}$ increases. For $i=i_{0}+k$, it reaches an extremum which is equal to the relative slope ratio of $m_{\text {left }}$ and $m_{\text {right }}$. For $i>i_{0}+k$, the function approaches zero again with $\Delta m_{\text {Ratio }}=0$ for $i>i_{0}+2 k$. Using this function, the task of

5 In both cases the gripper motion is restricted to linear motions because rotation generally influence the measured moment, and thus, may lead to the erroneous detection of transitions. 
detecting a slope change in $f$ is transformed into the task of detecting an extreme value of vector $\Delta m_{\text {Ratio }}{ }^{6}$

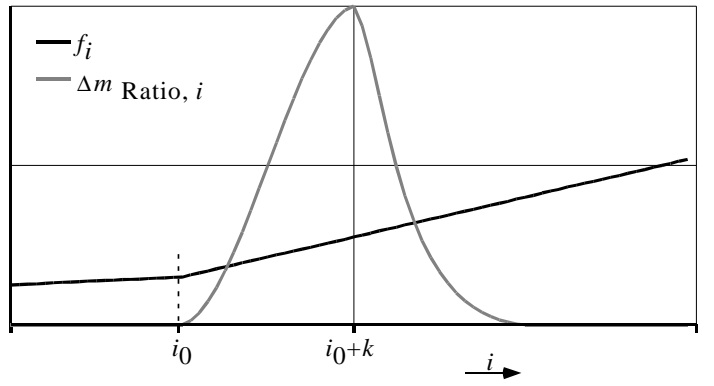

Figure 8: Discrete function $f_{i}$ consisting of two linear segments and relative difference $\Delta m_{\text {Ratio }}$ of the mean straight line

However, the course of $\Delta \boldsymbol{m}_{\text {Ratio }}$ has typically much more extrema than there are state transitions to be detected. Therefore, we use the following algorithm with some additional criteria. The vectors $\boldsymbol{m}_{\mathrm{MSL}}$ and $\Delta \boldsymbol{m}_{\text {Ratio }}$ are defined as given above, $b_{\mathrm{MSL}, i}$ is the intercept of the MSL in sample $i$, defining the MSL together with $m_{\mathrm{MSL}, i}$.

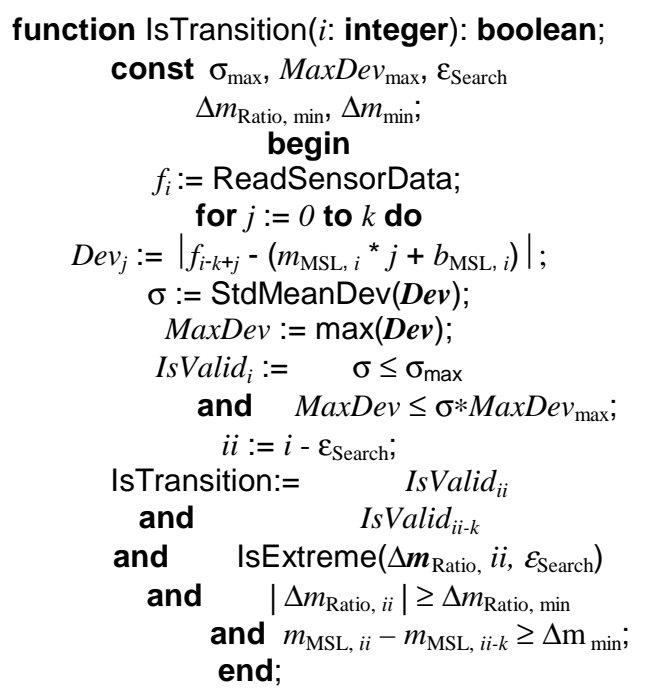

After sampling a new data value $f_{i}$ from the sensor, we compute the MSL given by the samples $i-k, \ldots i$. If the following two conditions are fulfilled, we suppose the sampled data to be valid $\left(\right.$ IsValid $_{i}:=$ true $)$ :

On the one hand, the sharpness of the extreme value is increased with $k$ decreasing. On the other hand, the susceptibility to distortions is increased, too. 
- the standard mean deviation $\sigma$ between the data values and the MSL is not greater than a threshold $\sigma_{\max }$, and

- the maximum deviation MaxDev from the MSL is not greater than the $\operatorname{MaxDev}_{\max }$-fold of $\sigma$.

Otherwise, the data are considered to be corrupted by noise or other distortions $\left(\right.$ IsValid $_{i}:=$ false).

After performing this check, $\Delta m_{\text {Ratio, } i}$ is computed as described above. Please note that the algorithm is based on a search for extreme values of $\Delta \boldsymbol{m}_{\text {Ratio}}$. Therefore, a state transition in sample $i_{0}$ can not be detected immediately, but only in sample $i_{0}+k+\varepsilon_{\text {Search }}$, with $2 \varepsilon_{\text {Search }}$ being the width of the window used for the extremum search. (In the opposite to the handling of rigid materials, a delayed detection of state transitions is less critical for deformable objects.) Therefore, the transition detection is performed for sample $i i=i$ - $\varepsilon_{\text {Search }}$ in step $i$.

For a state transition, the value sampled in step ii must fulfill the following conditions:

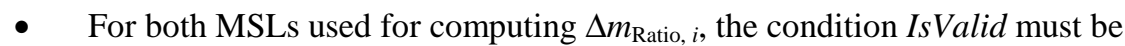
fulfilled. That is, the regarded area of the data array must not be severely disturbed.

- The function vector $\Delta \boldsymbol{m}_{\text {Ratio }}$ must suppose an extremum at position $i i$ as discussed above.

- Both the absolute value of $\Delta m_{\mathrm{Ratio}, i i}$ (that is, the relative slope difference of the MSLs) and the absolute slope difference $\Delta m_{i i}$ must be greater or equal than given threshold values $\Delta m_{\text {Ratio, min }}$ and $\Delta m_{\text {min }}$, respectively.

Figure 9 clarifies these conditions for a $\mathrm{N} \rightarrow \mathrm{V} / \mathrm{F}$ transition, using the absolute value of the moment vector $\boldsymbol{M}$ as measuring signal.

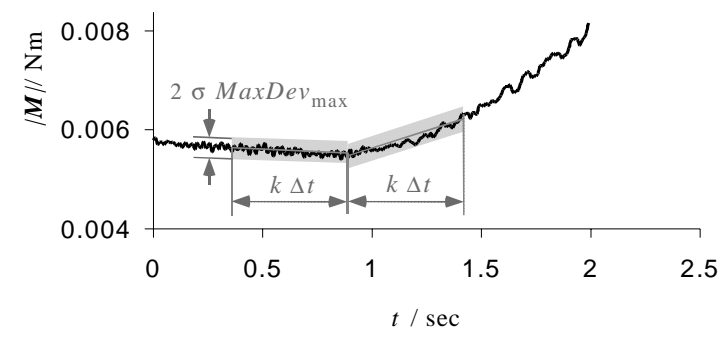

Figure 9: Absolute value of moment $\boldsymbol{M}$ for a transition $\mathrm{N} \rightarrow \mathrm{E} / \mathrm{E}$

In the formulation given above, the algorithm considers only one case of all possible slope changes. According to some above assumptions, the slope of the considered function may be either positive, negative, or zero for both $t<t_{0}$ and $t>t_{0}$. Therefore, the algorithm must be extended. The algorithm given above can be adopted to the detection of all kinds of transitions by considering the signs of $\Delta m_{\text {Ratio }}$ and $\Delta m$. 
For detecting oscillations $\mathbf{P}(\boldsymbol{a}, \boldsymbol{b}, \boldsymbol{c})$, we have to remind the fact that none of its characteristics, i.e., frequency, amplitude and damping, are generally known in advance. Thus, an algorithm is required for the robust on-line detection of arbitrary (sinusoidal) oscillations.

Our detection algorithm is based on the following assumptions:

1. though the oscillation period $T$ is unknown, we can give a lower boundary $T_{\min }$ with $T \geq T_{\min }$ (and possibly also an upper boundary $T_{\max }$ ).

2. though both the (initial) amplitude $A_{0}$ and damping are unknown, we can give a lower amplitude boundary $A_{\min }$ and a number $n_{\min }$ with $A \geq A_{\min }$ for at least $n_{\min }$ consecutive oscillation extrema.

For the detection, we perform an online search for extreme values in the force or moment function. An oscillation is detected when there are $n_{\min }$ consecutive extrema, each having a temporal distance of at least $T_{\min }$ and an elongational distance of at least $A_{\min }$ to the preceding one, as shown in Figure 10 for $n_{\min }=3$. Though both the oscillation amplitude and the period depend on the workpiece and the situation, the algorithm proved to work reliably for a large variety of workpieces and situations without changing $A_{\min }$ and $T_{\min }$.

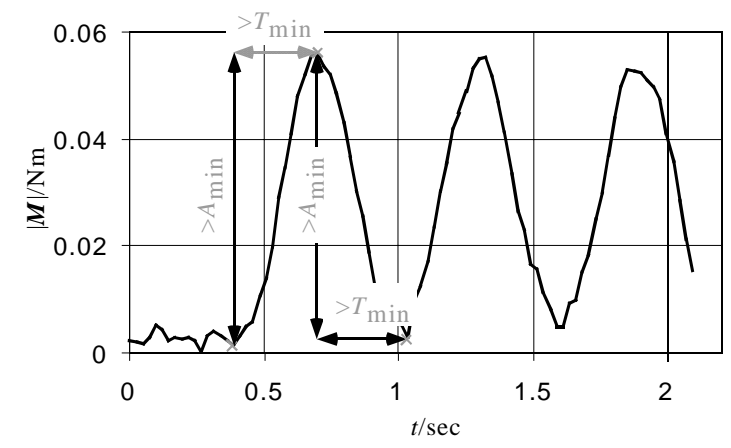

Figure 10: Amount of moment vector $\boldsymbol{M}$ for a $\mathrm{V} / \mathrm{F} \rightarrow \mathrm{V} / \mathrm{E} \rightarrow \mathrm{N}$ sequence. For clarity reasons, the static load due to gravity is not shown

\section{Experimental Results}

In this section, results of the sensor-based robot manipulation of deformable linear objects using the state transition recognition approach are presented. Both sensor systems are used: machine vision and force/torque measurement.

\subsection{Vision-based workpiece manipulation}

For the robot manipulation experiments, a Kuka KR15 robot is used. The robot controller executes motion commands sent from a Linux-PC with $350 \mathrm{MHz}$ Intel 
Pentium II Processor. As stationary camera, a standard video CCD-Camera (Hitachi KP M3) is used, and as hand camera a Teli CS $3710 \mathrm{C}$ is used. The data are sent by a standard frame grabber (Eltec PC-EYE I) to the vision processing computer which is a Linux-PC with either a $133 \mathrm{MHz}$ or a $350 \mathrm{MHz}$ Intel Pentium II Processor. As force torque sensor an experimental sensor of the "Deutsche Luft- und. Raumfahrtgesellschaft" (DLR) is used. The gripped workpiece is a pneumatic polyurethane wire with the outer diameter of $6 \mathrm{~mm}$ and a length of $300 \mathrm{~mm}$. The obstacle is a car door frame which is mounted in a horizontal lying position. Details of the software system and its structure can be found in [28].
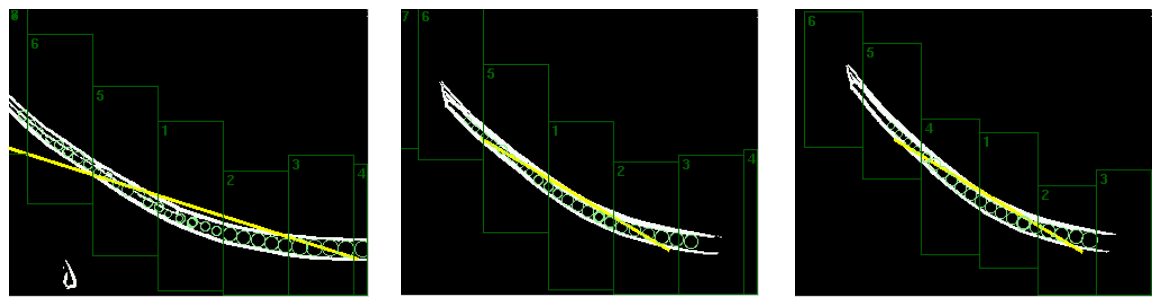

Figure 11: A sequence of images where a pneumatic wire is segmented and tracked with the hand camera

The segmentation and feature analysis algorithms were tested in various experiments and work well under both daylight and artificial lighting conditions. Measurements yield a mean execution time of $1.1 \mathrm{~ms}$ on an Intel Pentium machine with $133 \mathrm{MHz}$ processor when a workpiece is detected. For common situations and their well-known problems, usually a small set of parameters has to be adapted what can be done from an expert in a few minutes. Figure 11 shows a sequence of images where a pneumatic wire is segmented and tracked with the hand camera by using a gray value difference detection with the Sobel operator.

Since the force/torque sensor produces very similar feature data compared to both vision sensors, subsequently only experiments involved in the detection of a state transition with our force/torque sensor will be reported. Furthermore, the algorithms described here have been investigated for both isolated state transitions and an assembly task.

\subsection{Force/torque-based workpiece manipulation}

As robot manipulator, a KUKA KR 15 industrial robot with PC-based KR C1 robot controller has been used. As DLOs, we used a Polyurethane pneumatic wire with $6 \mathrm{~mm}$ outer and $4 \mathrm{~mm}$ inner diameter, a spring steal wire of $1 \mathrm{~mm}$ diameter, and a commercial spring steal ruler of length $0.5 \mathrm{~m}$ and $(18 \times 0.5) \mathrm{mm}^{2}$ cross section. Due to the bending rigidity of the objects, the ruler shows the highest contact forces, while they are smallest for the pneumatic hose. Accordingly, the difficulty of detecting state transitions increases in this order, too. 
As an example, Figure 9 shows the absolute value of moment $\boldsymbol{M}$ for a sequence $\mathrm{N} \rightarrow \mathrm{E} / \mathrm{F} \rightarrow \mathrm{E} / \mathrm{E}$ with motion direction $\mathrm{MD}_{1}$ according to Figure 3 for the steal ruler and the hose. Though the contact force is considerably higher for the steal ruler, the transitions were detected correctly for both materials with adopting only the parameter $\Delta m_{\min }$. Compared to the steal ruler, the detection of the $\mathrm{N} \rightarrow \mathrm{E} / \mathrm{F}$ transition is critical for the hose because the force caused by the E/F-contact is very small. In such cases, the reliability can be considerably improved if the time of the state transition is approximately known. If this condition is fulfilled, erroneous detections of transitions can be avoided by starting the detection algorithm only some time before the detection. Especially when starting and stopping gripper motions, the detection of state transitions should not be active.
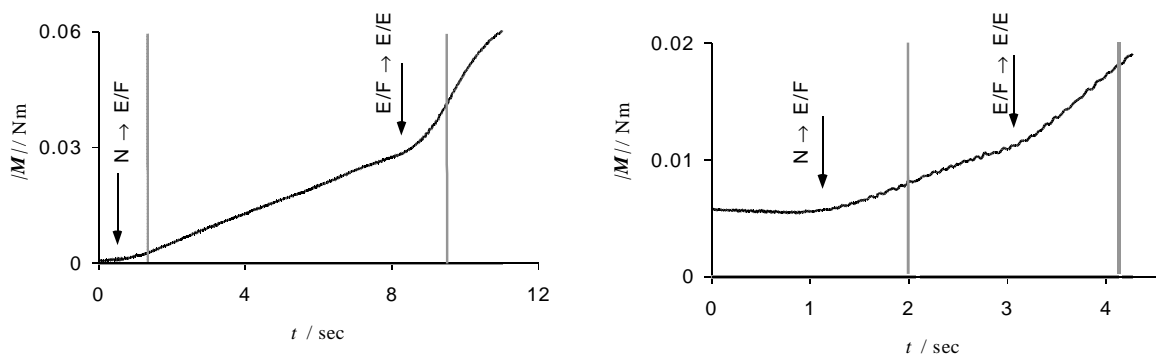

Figure 12: Transition sequence $N \rightarrow E / F \rightarrow E / E$ for steal ruler (left) and pneumatic hose (right), using the following parameters: Sampling period $\Delta t=10 \mathrm{~ms}, k=80$ samples, $\Delta m_{\min }=1 * 10^{-5} \mathrm{Nm}$ (ruler) respectively $\Delta m_{\min }=2 * 10^{-5} \mathrm{Nm}$ (hose), $\Delta m_{\text {Ratio, min }}=0.5$,

$$
\sigma_{\max }=4 * 10-5 \mathrm{Nm}, \varepsilon_{\text {Search }}=15 \text { samples }
$$

Compared to the moment shown here, the force signal is often found to be rather noisy and could hardly be evaluated. This is due to the fact that the forces to be measured are rather small. However, because the distance between the gripper and the contact point is rather long, even these small forces cause significant moments. Especially all disturbances caused by the (rather high) inertia in the gripper impact on the force much more than on the moment.

In addition to the investigation of isolated transitions, we performed the task of inserting a pneumatic hose into a guiding groove as described in [21] and used the algorithm presented here for detecting the contact state transitions. The algorithm proved to be reliable in this task. However, we find that the detection is less critical for the transitions with the contact point being rather close to the gripper than for transitions with the contact point (point of force application) being far from the gripper. This is caused by the fact that the bending rigidity of the workpiece increases with decreasing length between the gripper and the contact point. Especially for low contact forces, some problems are caused by friction which may be of larger influence than the change of contact force. Thus, the friction coefficient between the workpiece and the obstacle should be as small as possible. 


\section{Conclusions and Future Work}

In the assembly of rigid workpieces, the consideration of contact states and state transitions has been proven to be a suited method for creating robust manipulation routines for multiple purposes. We expect that this is valid for deformable workpieces as well. In this paper, we investigate contact states and state transitions of deformable linear objects in a rigid environment. The application to a typical industrial problem shows that this principle can be easily used for describing assembly tasks.

In this chapter, a new and systematic approach to the machine vision-based and force/torque-based robot manipulation of deformable linear workpieces is proposed. This powerful approach reduces the computational needs by using a simple state-oriented model of the workpiece.

The states describe the relation between the workpiece and an obstacle, and they are derived from the sensor data and the features delivered by two different sensor systems (vision and FTS). Using the vision sensors, the workpiece image is segmented from a standard video frame using a new and fast segmentation algorithm. With the FTS, forces and moments can be measured directly. Finally, for both sensor systems, the workpiece features are computed in order to recognize state transitions during the manipulation of the workpiece by a robot.

Experimental results prove the applicability of our approach. Characteristic feature changes are derived from manual manipulation and observation experiments. They are used for implementation and optimization of the sensor processing and the workpiece state classification. Two state transitions that are recognized reliably with our system are presented.

Because there are only two main types of characteristic functions (oscillations and slope changes), two detection algorithms were developed for detecting the transitions. Both of them where found to be reliable in an experimental investigation. However, the robustness of the detection depends on several physical and technical parameters as for example the workpiece rigidity, friction coefficients, and the sensor resolution.

For future work, we need to further investigate on which parameters can be computed automatically and how can their number be minimized. We have to answer the question which sensor system is useful for which transition and under which conditions. This will also include a comparison of the results of our research for every sensor system. As further step, we will concatenate the implemented vision-based robot primitives for deformable linear objects in order to execute complex manipulation tasks.

The detection algorithms presented here contain some "hard" threshold values that need to be set according to the specific task. Additionally, the detection may fail in the presence of negligible uncertainties. Therefore, we propose that a fuzzification of the algorithms will improve both the generality and reliability of the state transition recognition. For improving the reliability when contact forces are small, we will investigate the usage of force/torque sensors with very high reso- 
lution. The next major step is to construct encapsulated routines for performing manipulation tasks, including both gripper motions and transition detection.

\section{References}

Byun J.-E., Nagata T.: "Determining the 3-D pose of a flexible object by stereo matching of curvature representations". In: Pattern Recognition: The Journal of the Pattern Recognition Society, vol. 29, no. 8, pp. 1297-1308, 1996.

Nakashima M., et al.: "Application of semi-automatic robot technology on hot-line maintenance work". In: IEEE Int. Conf. on Robotics and Automation, pp. 843-850, 1995.

Hirai, S., Wakamatsu, H., and Iwata, K.: "Modeling of deformable thin parts for their manipulation". In: Proc. 1994 Int. Conf. on Robotics and Automation (ICRA94), vol. 4, pp. 2955-2960, San Diego, USA, May 1994.

Wakamatsu, H., Hirai, S., and Iwata, K.: "Modeling of linear objects considering bend, twist and extensional deformations". In: Proc. 1995 Int. Conf. on Robotics and Automation (ICRA95), vol. 1, pp. 433-438, Nagoya, Japan, May 1995.

Wakamatsu H., et al.: "Dynamic analysis of rodlike object deformation towards their dynamic manipulation", In: Proc. 1997 IEEE/RSJ Int. Conf. on Intelligent Robots and Systems (IROS'97), pp. 196ff, Grenoble, France, September 1997.

Remde A., Henrich D.: "Direct and Inverse Simulation of Deformable Linear Objects". Chapter in this book.

Inoue H., Inaba M.: "Hand-eye coordination in rope handling". In: Proc. of the First Int. Symp. On Robotics Research, pp. 163-174, New Hampshire, USA, September 1983.

Zheng Y. F., Pei R. Chen C.: "Strategies for automatic assembly of deformable objects". In: Proc. 1991 Int. Conf. on Robotics and Automation (ICRA'91), vol. 3, pp. 2598-2630, Sacramento, USA, April 1991.

Nakagaki H., et al: "Study of insertion task of a flexible wire into a hole by using visual tracking observed by stereo vision". In: Proc. 1996 Int. Conf. on Robotics and Automation, vol. 4, pp 3209-3214, Minneapolis, MN, USA, April 22-28, 1996.

Kraus W., McCarragher B. J.: "Case studies in the manipulation of flexible parts using a hybrid position/force approach". In: Proc. 1997 Int. Conf. on Robotics and Automation (ICRAí97), vol. 1, pp. 367372, Albuquerque, USA, April 1997. 

one-dimensional objects by using vision sensors". In: Proc. of the 1991 IEEE Int. Conf. on Robotics \& Automation, Sacramento, California, USA, April 1991.

Nakagaki H., Kitagaki K., Ogasawara T., Tsukune H.: "Study of deformation and insertion tasks of a flexible wire". In: Proc. of IEEE Int. Conf. on Robotics and Automation (ICRA97), pp. 2397-2402, Albuquerque, USA, April 1997.

Smith P.W.: "Image-based manipulation planning for non-rigid objects". In: Proc. 1998 Int. Conf. on Robotics and Automation, vol. 4, pp 3540-3545, Leuven, Belgium, May 1998.

Hasegawa T., Suehiro T., Takase K.: "A model-based manipulation system with skill-based execution". In: IEEE Transactions on Robotics and Automation, vol. 8, no. 5, pp. 535-544, Oct. 1992.

Onda H., Hirukawa H., Takase K.: "Assembly motion teaching system using position/force simulator - Extracting a sequence of contact state transition". In: IEEE/RSJ International Conference on Intelligent Robots and Systems (IROS'95), vol. 1, pp. 9-16, 1995.

Crowell R. H., Fox R. H.: "Introduction to knot theory", Springer New York Heidelberg, 1977, ISBN 0-387-90272-4, 3-540-90272-4.

Suehiro T., Takase K.: "Representation and control of motion in contact and its application to assembly tasks". In: Proc. 5th Int. Symp. on Robotics Research, 1989.

Higashijima K., Onda H., Ogasawara T.: "Planning for wire obstacles avoidance using ultrasonic sensors". In: IEEE/RSJ Int. Conf. on Intelligent Robots and Systems (IROS'98), Victoria, October 1998.

Morrow J. D., Khosla P. K.: "Manipulation task primitives for composing robot skills". In: IEEE Int. Conf. on Robotics and Automation (ICRAí97), pp. 3354-3359, Albuquerque, USA, April 1997.

Henrich D., Ogasawara T., Wörn H. "Manipulating deformable linear objects - Contact states and point contacts". In: 1999 IEEE Int. Symp. on Assembly and Task Planning (ISATP'99), Porto, Portugal, July 21-24, 1999.

Remde A., Henrich D., and Wörn H.: "Manipulating deformable linear objects: Contact state transitions and transition conditions". In: 1999 IEEE/RSJ Int. Conf. on Intelligent Robots and Systems (IROS'99), Kyongju, Korea, October 1999.

Morris G. H., Haynes L. S.: "Robotic assembly by constraints". In: Proc. 1987 IEEE Int. Conf. on Robotics and Automation (ICRA'87), pp. 1507-1515, 1987. 
Remde, A., Henrich, D., and Wörn H.: "Manipulating deformable linear objects - Force based detection of contact state transitions -". Submitted to: 2000 IEEE International Conference on Robotics and Automation (ICRA 2000), San Francisco, CA, USA, April 2000.

Morrow J.D., Khosla P.: "Sensorimotor primitives for robotic assembly skills". In: Proc. 1995 IEEE Int. Conf. on Robotics and Automation, pp. 1894-1899, Nagoya, Japan, May 1995.

[25]

Hutchinson S., Hager G.D., and Corke P.I.: "A tutorial on visual servo control". In: IEEE Trans. on Robotics and Automation, vol. 12, no. 5, October 1996.

Abegg, F., Henrich, D., and Wörn H.: Manipulating deformable linear objects: Vision-based recognition of contact state transitions". In: Proc. $9^{\text {th }}$ Int. Conf. on Advanced Robotics (ICAR'99), Tokyo, Japan, Oct. 25-27, 1999.

Gray A.: "Modern differential geometry of curves and surfaces", CRC Press, 1994.

Henrich D. and Abegg F. and Wurll Ch. and Wörn: "A parallel control architecture for industrial robot cells". In: Proc. 4th International Symposium on Distributed Autonomous Robotic Systems, pp. 77-86, Karlsruhe, May 1998. 